\title{
Turbulent transport stabilization by ICRH minority fast ions in low rotating JET ILW L-mode plasmas
}

\author{
N. Bonanomi ${ }^{1,2}$, P. Mantica ${ }^{2}$, A. Di Siena ${ }^{3}$, E. Delabie ${ }^{4}$, C. Giroud ${ }^{5}$, \\ T. Johnson ${ }^{6}$, E. Lerche ${ }^{7}$, S. Menmuir ${ }^{5}$, M. Tsalas ${ }^{8,5}$, D. Van Eester ${ }^{7}$ \\ and JET contributors*
}

EUROfusion Consortium, JET, Culham Science Centre, Abingdon, OX14 3DB, UK

1) University of Milano-Bicocca, Milano, Italy

2) CNR - Plasma Physics Institute "P. Caldirola", Milano, Italy

3) Max Planck Institute for Plasma Physics, Boltzmannstr. 2, 85748 Garching, Germany

4) Oak Ridge National Laboratory

5) Culham Centre for Fusion Energy, Abingdon, OX14 3DB, UK

6) KTH Royal Institute of Technology, Stockholm, Sweden

7) LPP-ERM/KMS, TEC partner, Brussels, Belgium

8) DIFFER - Dutch Institute for Fundamental Energy Research, Eindhoven, The Netherlands

*See the author list of "X. Litaudon et al., 2017 Nucl. Fusion 57, 102001"

\begin{abstract}
The first experimental demonstration that fast ion induced stabilization of thermal turbulent transport takes place also at low values of plasma toroidal rotation has been obtained in JET ILW (ITER-Like Wall) L-mode plasmas with high $\left({ }^{3} \mathrm{He}\right)$-D ICRH (Ion Cyclotron Resonance Heating) power. A reduction of the gyro-Bohm normalized ion heat flux and higher values of the normalized ion temperature gradient have been observed at high ICRH power and low NBI (Neutral Beam Injection) power and plasma rotation. Gyrokinetic simulations indicate that ITG (Ion Temperature Gradient) turbulence stabilization induced by the presence of high-energetic ${ }^{3} \mathrm{He}$ ions is the key mechanism in order to explain the experimental observations. Two main mechanisms have been identified to be responsible for the turbulence stabilization: a linear electrostatic wave-fast particle resonance mechanism and a nonlinear electromagnetic mechanism. The dependence of the stabilization on the ${ }^{3} \mathrm{He}$ distribution function has also been studied.
\end{abstract}

\section{Introduction}

In JET C-Wall L-mode plasmas with high Neutral Beam Injection (NBI) power and with Ion Cyclotron Resonance Heating (ICRH) power in $\left({ }^{3} \mathrm{He}\right)-\mathrm{D}$ minority scheme, a strong stabilization of the turbulent ion heat transport has been observed in the region $R \lesssim 3.4 m(r / a \lesssim 0.4, a$ being the minor radius) [1, 2, 3]. The stabilization was observed experimentally as a reduction of the gyro-Bohm normalized ion heat flux and higher values 
of the normalized ion temperature gradient, and was associated with an upshift of the Ion Temperature Gradient (ITG) modes critical gradient and with a significant reduction of the ion stiffness (i.e. the rate of increase of the gyro-Bohm normalized ion heat flux in response to an increase in $\left.R / L_{T i}=-R \nabla T_{i} / T_{i}\right)$. Gyrokinetic simulations indicated that the key stabilization mechanism acting on ITG turbulence was a nonlinear electromagnetic mechanism enhanced by the fast ion (D and $\left.{ }^{3} \mathrm{He}\right)$ pressure [4,5]. Gyrokinetic simulations predict an important reduction of the linear growth rate of ITG modes when considering fast ions and electromagnetic effects and a nonlinear enhancement of these effects. An important role of nonlinear electromagnetic stabilization of ITG turbulence in plasma with high $\beta$ has been found in [6], and a strong nonlinear electromagnetic stabilization of the ion heat transport has been predicted for JET and ASDEX Upgrade H-modes with high plasma $\beta$ [7, 8]. Recent results [9] indicate that an electrostatic mechanism, related related to a wave-fast particle resonant interaction $[10,11]$, can also play an important role in the stabilization of ITG modes in some conditions. The study in ref. [9] predicts also a more important role of fast ${ }^{3} \mathrm{He}$ with respect to fast $\mathrm{D}$ for the stabilization of ITG modes in the experimental conditions studied in $[2,4]$, the difference being related to the different values of the temperatures and of the normalized density $\left(R / L_{n}\right)$ and temperature $\left(R / L_{T}\right)$ gradients of the two different fast ion species. In particular high values of $\left(R / L_{T}\right) /\left(R / L_{n}\right)$ are expected to be favorable for the stabilization, while the optimal fast ion temperature for the resonance with the drift waves depends on the fast ion mass. Further mechanisms, related to ITG mode stabilization caused by main ion dilution due to the presence of fast ions, or to changes in effective plasma parameters, have been proposed for for ASDEX Upgrade low density plasmas with high NBI heating [12] or for JET L-mode plasmas with high NBI heating [13].

Experimentally, all the plasmas where the strong turbulent ion heat transport stabilization by fast ions has been observed so far featured high NBI power. High NBI leads to high toroidal plasma rotation, that can also induce a strong ITG turbulence stabilization [14, 15]. It is therefore difficult, when using high NBI power, to separate experimentally the effects of plasma rotation and the effects related to fast ions, and one has to rely on modelling to quantify the impact of the two stabilizing effects, as done in [4, 5]. In ASDEX Upgrade, a study on discharges with $4.5 \mathrm{MW}$ of NBI power and with 0 or $3.5 \mathrm{MW}$ of ICRH power in $\left({ }^{3} \mathrm{He}\right)-\mathrm{D}$ minority scheme showed that fast ${ }^{3} \mathrm{He}$ ions were necessary, in the gyrokinetic simulations, in order to explain the strong reduction of the ion heat flux and the strong increase of the ion temperature peaking observed experimentally [16]. In DIII-D, using co-and counter-NBI, a reduction of the ion heat flux was obtained in absence of plasma rotation [17], but other parameters, such as $T_{e} / T_{i}$ (known to have a strong effect on ITG modes [18, 19]), were changing between the plasmas. Furthermore, a detailed study of the effects of fast ions is missing for this case. So far, no experimental evidence of a strong stabilization of the turbulent ion heat flux, due to fast ions, has been obtained in absence, or with low, plasma rotation and with no substantial changes in other important parameters such as $s / q, T_{e} / T_{i}$. In this work, we present the first experimental evidence, in JET ILW L-mode plasmas with low plasma rotation, of a strong reduction of the turbulent thermal transport related to an increase of fast ${ }^{3} \mathrm{He}$ ion energy density, while keeping the other relevant plasma parameters constant. This is a direct experimental evidence that these stabilization mechanisms are effective also at low plasma rotation, which is an important element for the extrapolation of these effects to ITER scenarios. Nonlinear gyrokinetic simulations indicate that the stabilization of ITG turbulence induced by the presence of fast ${ }^{3} \mathrm{He}$ ions is the key mechanism to reproduce the experimental observations. In the cases presented here, unlike in those presented in [2, 4], besides the electromagnetic effects, also a significant role of the electrostatic effects related to fast ${ }^{3} \mathrm{He}$ ions is found in the simulations. The dependence of both effects on the ${ }^{3} \mathrm{He}$ distribution function has also been studied and found to be significant.

The paper is organized as follows: in section 2 the experimental set-up is described and in section 3 the gyrokinetic simulation set-up is presented. In section 4 the experimental results are described, while in section 5 the gyrokinetic simulations results are shown and compared to the experiment. The conclusions are reported in section 6 . 


\section{Experimental set-up and methods}

The discharges studied in this paper were made in the JET tokamak (major radius $R_{0}=2.96 \mathrm{~m}$, minor radius $a=1 \mathrm{~m}$ ) with ILW and are D plasmas with vacuum toroidal magnetic field $B_{T} \approx 3.3 T$, plasma current $I_{p} \approx 2 M A$ and safety factor at the flux surface that contains the $95 \%$ of the poloidal flux $q_{95} \approx 5$. The heating power consists of $2.6-7 \mathrm{MW}$ of ICRH and of $1.7 \mathrm{MW}$ of NBI. The ICRH power is deposited on ${ }^{3} \mathrm{He}$ minority ions and collisionally transferred to thermal D ions. Using a ${ }^{3} \mathrm{He}$ concentration $\left[{ }^{3} \mathrm{He}\right] \approx 9 \%$ ensures dominant thermal ion heating [20], The ICRH power was deposited on-axis $(R \approx 3.0 \mathrm{~m}, r / a \approx 0)$ in order to obtain the maximum heating power density and energy density of ${ }^{3} \mathrm{He}$ fast ions in the centre of the plasma (see figure 1 ), where the magnetic shear, known to have a strong effect on the stabilization of ITG modes by fast ions [4, 9], is minimum. Fixing the NBI heating power to the minimum value for Charge Exchange (CX) measurements and using different levels of ICRH power (and so different levels of fast ${ }^{3} \mathrm{He}$ energy density), a study of the effects of fast ${ }^{3} \mathrm{He}$ ions on the plasma thermal transport, while keeping the plasma rotation and the fast $\mathrm{D}$ population low, was possible. The ICRH power deposition and the ${ }^{3} \mathrm{He}$ fast ions density, energy density and distribution function have been evaluated using the SELFO code [21, 22]. Figure 1 shows the comparisons of the time evolution of the heating powers (figure 1a), of the profiles of the ICRH power deposition on thermal ions and of the fast ${ }^{3} \mathrm{He}$ energy densities (figure 1b), between a discharge with $2.6 \mathrm{MW}$ of ICRH (n. 90671) and a discharge with $7 \mathrm{MW}$ of ICRH (n. 90672). The NBI heating power on electrons and on ions and the fast D pressure in the plasma have been calculated with the PENCIL code [23]. The fast D energy density has been found to be negligible compared to the fast ${ }^{3} \mathrm{He}$ energy density in discharges with high ICRH power. The ohmic power density has been calculated using $P_{O h m}=\eta \cdot j^{2}$, where $\eta$ is the resistivity of the plasma and $j$ is the plasma current density reconstructed by the EFIT equilibrium code constrained by polarimeter measurements [24]. Also the exchanged power density between ions and electron, $p_{e i}[25]$, has been calculated analytically. The radiated power density $p_{\text {rad }}$, calculated from bolometry, has been found to be negligible inside $R \approx 3.8 m(r / a \approx 0.8)$. The powers $P_{O H M}, P_{I C R H}, P_{N B I}, P_{e i}$ and $P_{r a d}$ have been obtained integrating on the plasma volume within a flux surface and the ion and electron heat fluxes are expressed in gyro-Bohm units as

$$
\begin{aligned}
q_{i, g B_{i}} & =\left[\left(P_{I C R H, i}+P_{N B I, i}+P_{e i}\right) / \Sigma\right] \cdot R_{0}^{2} /\left(n_{e} T_{i} \rho_{i}^{2} v_{t h, i}\right) \\
q_{e, g B_{s}} & =\left[\left(P_{I C R H, e}+P_{N B I, e}+P_{O H M}-P_{e i}-P_{r a d}\right) / \Sigma\right] \cdot R_{0}^{2} /\left(n_{e} T_{e} \rho_{s}^{2} c_{s}\right)
\end{aligned}
$$

where $\Sigma$ is the area of the considered flux surface, $c_{s}=\sqrt{T_{e} / M_{i}}, v_{t h, i}=\sqrt{T_{i} / M_{i}}, \rho_{i}=v_{t h, i} M_{i} / e B_{T}$ and $\rho_{s}=c_{s} M_{i} / e B_{T}$. Typical errors on the heat fluxes are $\sim 20 \%$. For the measurement of the electron temperature $T_{e}$ the ECE (Electron Cyclotron Emission) diagnostic has been used, while the ion temperature $T_{i}$ and the plasma toroidal rotation $\Omega_{t}$ have been measured by the CX diagnostic. For the charge exchange measurements, Be and Ne impurities have been used $(\sim 0.1 \%$ of Ne has been puffed into the plasma in order to improve the CX measurements). Furthermore, a charge-exchange measurement recently made available at JET, that uses beam modulation for background subtraction and fits all active line features to extract the deuterium charge exchange line [26, 27], has been used and will be indicated as $D_{\alpha} \mathrm{CX}$. The density profiles of Be, Ne and thermal ${ }^{3} \mathrm{He}$ have been measured using the CX diagnostic [28]. The electron density $n_{e}$ has been measured by high-resolution Thomson scattering (HRTS). The radial profile of the safety factor $q$, of the magnetic shear $s=r / q \cdot d q / d r$ and of the equilibrium plasma geometry have been reconstructed by the EFIT equilibrium code constrained by polarimeter measurements. Local values of $R / L_{T i}=-R_{0} \nabla T_{i} / T_{i}, R / L_{T e}=-R_{0} \nabla T_{e} / T_{e}$ and $R / L_{n}=-R_{0} \nabla n_{e} / n_{e}$ are obtained by local linear fits of $\ln \left(T_{i}\right), \ln \left(T_{e}\right)$ and $\ln \left(n_{e}\right)$ radial profiles averaged over a time interval $\Delta t \approx 1 \mathrm{~s}$. The fits are done using $r=\left(R-R_{i n}\right) / 2, R$ and $R_{\text {in }}$ being the outer and inner radii of the flux surface on the magnetic axis plane, and averaging other multiple fits using a variable number of data points around the chosen radius (3-9 points). Errors on these quantities are typically $\sim 15-20 \%$. 
The data analysis has been carried out at $\rho_{\text {tor }}=\sqrt{\left(\Phi / \pi B_{T}\right) /\left(\Phi / \pi B_{T}\right)_{\max }}=0.25(R \approx 3.24 \mathrm{~m}, r / a \approx 0.26)$ and $t \approx 6 s, \Phi$ being the toroidal magnetic flux. At this radial position and at this time, the changes in $q_{i, g B_{i}}\left(R / L_{T i}\right)$ have been observed to be the largest. No magnetohydrodynamic instabilities such as sawteeth or other large-scale instabilities, that could interfere with the analysis, have been observed at the radial position and at the time chosen for the analysis.

\section{Gyrokinetic simulation set-up}

Nonlinear gyrokinetic simulations, that calculate the turbulent part of the heat flux, have been carried out using the GENE (Gyrokinetic Electromagnetic Numerical Experiment) [29, 30] code and compared with the experiment. Linear gyrokinetic simulations have also been carried out, in order to study the effects of the changes in the plasma parameters on the linear growth rate of the main micro-instabilities. GENE solves the gyrokinetic Vlasov equations [31,32] coupled with the Maxwell equations within a $\delta f$ approximation and using field aligned coordinates $\{x, y, z\}$, where $z$ is the coordinate along the magnetic field line, $x$ is the radial coordinate and $y$ is the binormal coordinate.

In all the simulations, which are carried out in the local limit $(1 / \rho * \sim 400)$, Miller geometry [33] has been used as well as collisions, external flow shear $\gamma_{E \times B}$ (even if very low in our case), kinetic D ions, kinetic electrons and kinetic ${ }^{3} \mathrm{He}$ ions (considering $n_{3}{ }_{\mathrm{He}} / n_{e}=9 \%$ ). Finite- $\beta$ effects (considering both $B_{\perp}$ and $B_{\|}$ fluctuations) have been considered in some simulations. For ${ }^{3} \mathrm{He}$, a Maxwellian distribution function has been used in most simulations. However, when considering highly energetic, non thermalized, ${ }^{3} \mathrm{He}$ particles, the Maxwellian distribution function is not adequate to describe the real distribution function of this species and the results can be affected by this approximation [10, 34, 35]. For this reason, a new version of the GENE code [36, 37] able to use a numerical distribution function for ${ }^{3} \mathrm{He}$ (calculated using the SELFO code), has been used for few simulations. Typical grid parameters in the nonlinear simulations were as follows: perpendicular box sizes $\left[L_{x}, L_{y}\right] \approx[190,125] \rho_{s}$, phase-space grid discretization $\left[n_{x}, n_{y}, n_{z}, n_{v \|}, n_{\mu}\right]=[256,48,34,32,24-48]$ and $0.05 \lesssim k_{y} \rho_{s} \lesssim 2.4$. Detailed convergence tests have been carried out to choose these settings. The plasma parameters used as input in the simulations have been taken from discharge n. 90672 at $\rho_{\text {tor }}=0.25$ and $t \approx 6 \mathrm{~s}$. Some important values used in the simulation, when considering fast ${ }^{3}$ He particles, are: $Z_{\text {eff }}=$ 1.36, $q=1.4, R / L_{n,{ }^{3} \mathrm{He}}=R / L_{n e}=2.6, R / L_{T e}=8, R / L_{T, D}=7.2, R / L_{T,{ }^{3} \mathrm{He}}=15, T_{3} \mathrm{He}=12 T_{e}, T_{i} / T_{e}=$ $0.8, \beta_{\text {ref }}=8 \pi n_{e} T_{e} / B_{0}^{2} \approx 0.004, \nu *=\nu_{e i} \frac{q R_{0}}{\epsilon^{1.5} v_{t h, e}}=0.06, n_{3} \mathrm{He}_{e}=0.09 n_{e}, \gamma_{E x B}=-r / q \partial \Omega_{t} / \partial r R_{0} / c_{s}=0.003$, where $\epsilon=r / R_{0}, \nu_{e i}=\sqrt{2} \pi Z^{2} e^{4} n_{i} \ln (\Lambda) /\left(\sqrt{m_{e} T_{e}^{1.5}}\right), \ln (\Lambda)$ being the Coulomb logarithm, and $v_{t h, e}=\sqrt{T_{e} / m_{e}}$ . When not considering the fast ${ }^{3} \mathrm{He}$ ions in the simulations, we use $T_{3} \mathrm{He}_{e}=T_{i}$ and $R / L_{T^{3} \mathrm{He}}=R / L_{T i}$ without changing any other input parameter. We remark that in all the simulations $9 \%$ of ${ }^{3} \mathrm{He}$ is considered. What is changed between simulations with and without fast ${ }^{3} \mathrm{He}$ ions are the values of the ${ }^{3} \mathrm{He}$ temperature, of the ${ }^{3} \mathrm{He}$ temperature gradient and, in some cases, the ${ }^{3} \mathrm{He}$ distribution function. In the electrostatic simulations, a value of $\beta_{\text {ref }}=10^{-5}$ is imposed without changing any other input parameter. In the simulation of the low ICRH power case, the same parameters have been used except the reference $T_{e}$ value (lowered from $3.6 \mathrm{keV}$ to $2.6 \mathrm{keV})$, considering electromagnetic effects and just thermal ${ }^{3} \mathrm{He}$. Also a few electron-scale simulations have been performed, in order to study the impact of fast ions on ETG modes in nonlinear simulations. In these simulations the range $3 \leq k_{y} \rho_{s} \leq 96$ has been used and, as the linear growth rate of ETG modes does not strongly depend on the fast ions distribution function (see figure 4c), a Maxwellian distribution function has been used for the ${ }^{3} \mathrm{He}$.

The effect of other impurities $(\sim 1.2 \% \mathrm{Be}, \sim 0.3 \% \mathrm{Ne}, \sim 0.05 \% \mathrm{Ni})$ has been studied using linear gyrokinetic simulations and found to be negligible compared to the effect of ${ }^{3} \mathrm{He}$ in our case. Considering that, as shown by the CX measurements, these impurities are present in all the discharges in the same concentrations and with the same density peaking [28], they cannot explain the differences observed between discharges with low and high 
ICRH power. The difference in $N i$ concentration is $\sim 0.05 \%$, but, as discussed in section 5 , it has a negligible effect on ITG modes as it causes little main ion dilution. For these reasons, and in order to save computational resources, we decided to not include $B e, N e$ and $N i$ impurities in our simulations.

The nominal experimental value of the magnetic shear is $s=0.25$. Using this value, in the electromagnetic simulations with a Maxwellian distribution function for ${ }^{3} \mathrm{He}$, instabilities are present at $k_{y} \rho_{s} \lesssim 0.1$ (Kinetic Ballooning Modes (KBM)/energetic particles modes) when fast ${ }^{3} \mathrm{He}$ ions are considered. These modes lead to levels of ion heat flux in the nonlinear simulations not comparable with the experiment, as found also in [5, 8]. For these reason, when using the Maxwellian distribution function for the fast ions, the value of $s=0.46$ has been chosen, within experimental error bars, in order to avoid the presence of the low- $k_{y}$ instabilities. When using the SELFO numerical distribution function for the fast ions, these modes disappear also at $s=0.25$ and few simulations have been performed with this value.

\section{Experimental observations}

Although the ${ }^{3} \mathrm{He}$ concentration of $9 \%$ was used to maximize ion heating, in the high ICRH power cases SELFO simulations predict that a substantial part of the ICRH power is still absorbed by electrons due to the high energies achieved by the minority fast ions at such power levels. Both the ion and electron temperature profiles then show a general increase when ICRH power is increased. In addition, inside $\rho_{\text {tor }} \approx 0.35$, an important increase of $R / L_{T i}$ and strong reduction of $q_{i, g B_{i}}$ are observed in discharges with high ICRH power (figures $2 \mathrm{a}$ and $2 \mathrm{~b}$ ), while the values of $R / L_{T e}$ and of $q_{e, g B_{s}}$ are not changing (figure 8). In order to study what is causing these changes in $q_{i, g B}\left(R / L_{T i}\right)$, a comparison between discharges n. 90668 and n. 90671 (with $1.7 \mathrm{MW}$ of NBI and 2.6 MW of ICRH) and discharges n. 90670 and n. 90672 (with 1.7 MW of NBI and $6-7 \mathrm{MW}$ of ICRH) has been done at $t=6 \mathrm{~s}$ and at $\rho_{\text {tor }}=0.25$, as already explained in section 1 . In figure $2 \mathrm{a}$ the comparison of the $T_{i}$ radial profiles of discharges n. 90672 and n. 90671 (discharges n. 90668 and n. 90670 show similar behavior) is shown, while the comparison of the experimental $q_{i, g B_{i}}\left(R / L_{T i}\right)$ values is shown in figure $2 \mathrm{~b}$. The increase in the peaking of $T_{i}$, inside $\rho_{t o r} \approx 0.35$, and the strong reduction of $q_{i, g B}$ in discharges with high ICRH power are well visible outside error bars. The radial profiles of $T_{e}, n_{e}, s, q, \Omega_{t}$ for the same discharges are shown in figure 3. No substantial differences in these parameters are observed, except for higher values of $T_{e}$. Furthermore, $s / q, T_{e} / T_{i}, R / L_{n}, \Omega_{t}, \partial \Omega_{t} / \partial r$, that are known to have an important impact on turbulent thermal transport $[14,15,18,19,38,39,40]$, as well as light impurity density $\left(n_{3 \mathrm{He}} / n_{e} \approx 9-11 \%, n_{\mathrm{Be}} / n_{e} \approx 1.2 \%, n_{\mathrm{Ne}} / n_{e} \approx 0.3 \%\right)$ and light impurity density peaking (also known to have an impact on turbulent thermal transport [41, 42, 43, 44]) are similar, within error bars, over the whole radial profile, between discharges with low and high ICRH heating. $Z_{\text {eff }}$ is changing from $\sim 1.6$, in discharges with low ICRH, to $\sim 2$, in discharges with high ICRH power. The change in $Z_{\text {eff }}$ is mainly due to a medium $Z$ impurity $(\mathrm{Ni})$ coming from the ICRH antenna facilities. A change in the Ni concentration from $\sim 0.02 \%$ to $\sim 0.07 \%$ is enough to explain the change in $Z_{\text {eff }}$.

The main difference, between the plasmas with high and low ICRH heating power, is the fast ${ }^{3} \mathrm{He}$ energy density (figure $1 \mathrm{~b}$ ) inside $\rho_{\text {tor }} \lesssim 0.4$. The ${ }^{3} \mathrm{He}$ pressure modifies also the total plasma pressure $\left(p=\sum_{s} n_{s} T_{s}\right.$, the sum being over all the species in the plasma), as can be seen in figure $3 \mathrm{~d}$, and with the higher $T_{i}$ and $T_{e}$ temperatures, increases the plasma $\beta=8 \pi p / B_{0}^{2}$ in discharges with high ICRH heating. These changes are expected to lead to a strong stabilization of ITG modes and of the related turbulent ion thermal transport $[4,6,7,8,9,16]$.

\section{Gyrokinetic simulations}

As discussed in section 4, the main differences between discharges with low and high ICRH heating power, inside $\rho_{\text {tor }} \approx 0.4$, are the increase, at high ICRH power, of the plasma pressure $p$ and of the local plasma 
pressure gradient (related to a strong increase of the energy density of the fast ${ }^{3} \mathrm{He}$ ions) and the increase of $Z_{\text {eff }}$, related to the presence of $N i$ coming from the ICRH antenna facilities. The changes in the plasma pressure lead to changes in the plasma $\beta$ and in $\alpha_{M H D}=-q^{2} R(d \beta / d r)$. Both these parameters can have an impact on the plasma micro-instabilities. Higher $\beta$ can induce a stabilization of ITG modes (both linear [45, 46] and nonlinear $[4,7,8])$ and, over a certain limit, a destabilization of electromagnetic instabilities such as the Kinetic Ballooning Modes (KBM) [47, 48]. Increasing $\alpha_{M H D}$ can induce a stabilization of ITG modes through a geometric effect related to an increase of the Shafranov shift [49].

The analysis with gyrokinetic simulations, carried out as described in section 3 , focuses on these aspects. In all the linear gyrokinetic simulations, the nature of the main micro-instability is determined by the real part of the frequency (not shown in the figures).

\subsection{Linear gyrokinetic simulations}

\subsubsection{Effects of the changes in $Z_{\text {eff }}$ and $\alpha_{M H D}$}

The effects due to a change of $0.05 \%$ in the Ni concentration have been found negligible (reduction of $\gamma_{I T G}$ of $\sim 4 \%$ ) compared to the effects related to ${ }^{3} \mathrm{He}$, the $\mathrm{Ni}$ concentration being too low and the main ion dilution minimal. Also the effects related to the changes in $\alpha_{M H D}$ have been found to be minimal in our case. For these reasons, the value of $\alpha_{M H D}$ has been fixed, in all the simulations, equal to the one obtained considering the fast ${ }^{3} \mathrm{He}$, while Ni has not been considered in the simulations.

\subsubsection{Effects of fast ions on ITG modes}

Figure 4a shows the normalized linear growth rates of ITG modes, which are the dominant modes for $k_{y} \lesssim 1$, obtained from simulations with and without the electromagnetic effects and the fast ${ }^{3} \mathrm{He}$ ions. In the same figure, also the results obtained using the numerical distribution function ('SELFO') for ${ }^{3} \mathrm{He}$ are shown. At $0.2 \leq k_{y} \rho_{s} \leq 0.4$, where the major part of the ion heat flux is carried in the nonlinear simulations, a little stabilization, or a little destabilization, depending on $k_{y}$, of the ITG modes growth rate is obtained when considering electromagnetic (finite- $\beta$ ) effects but not the fast ${ }^{3} \mathrm{He}$ ions. When adding the fast ${ }^{3} \mathrm{He}$ ions, a strong stabilization of $\gamma$ is visible. The stabilization is due to electrostatic (reduction by $\sim 25 \%$ of $\gamma$ ) and electromagnetic (further reduction of $\gamma$ by $\sim 15 \%$ ) effects, with a total reduction of the linear growth rate by $\sim 40 \%$. When considering the numerical distribution function for ${ }^{3} \mathrm{He}$, this reduction is lower with respect to the one obtained considering a Maxwellian distribution function, but it is still strong $(\sim 25 \%)$. Using $s=0.25$, the same relative ITG growth rate reduction as for the case $s=0.46$ is obtained, but the values of $\gamma$ are lower with respect to the ones obtained using $s=0.46$ (figure $4 \mathrm{~b}$ ). Furthermore, using $s=0.25$, electromagnetic instabilities (KBM/energetic particle modes) appear at $k_{y} \lesssim 0.1$, causing a strong enhancement of the heat fluxes in the nonlinear simulations, not compatible with the experiment. Interestingly, when considering the numerical distribution function for ${ }^{3} \mathrm{He}$, these modes disappear and the nonlinear simulations give results comparable with the experiment.

As done in ref. [9], the contribution of the different species to the overall micro-instability growth rate has been investigated in velocity space through the energy conservation property of the Vlasov-Poisson equations $[50,51,52]$. In figure 5 the results obtained for the thermal $\mathrm{D}$ and ${ }^{3} \mathrm{He}$ are shown for the cases $T_{3_{H e}}=T_{i}$, $T^{T_{3}}=4 T_{e}$ (low ICRH power) and $T_{3} H_{e}=12 T_{e}$ (high ICRH power). The background distribution function of each species has been considered as an equivalent Maxwellian distribution. In the plots the position of the resonance, calculated with the reduced Vlasov model of [9], is also shown (black dotted lines). Note that for the case of thermal Deuterium the reduce model gives only a rough estimate of the resonance position. As can be seen, the resonance position is shifted to lower values of the velocity as the ${ }^{3} \mathrm{He}$ temperature is increased. While the contribution of $\mathrm{D}$ to the growth rate is always positive, the contribution of ${ }^{3} \mathrm{He}$ to the growth rate 
in the case $T_{3} H_{e}=12 T_{e}$ is dominated by the stabilizing region of velocity space, leading to a strong reduction of the growth rate. This change in the ${ }^{3} \mathrm{He}$ contribution to the growth rate is reflected also in the nonlinear simulations, where a negative flux is found for ${ }^{3} \mathrm{He}$ for $T_{3} \mathrm{He}=12 T_{e}$ (see section 5.2 and figure $6 \mathrm{~b}$ ). These results strongly support the role of the electrostatic fast ${ }^{3} \mathrm{He}$-drift wave resonance mechanism in the stabilization of the ITG modes observed in our simulations.

\subsubsection{Effects of fast ions on high-k instabilities}

In figure 4c, the effects of fast ions and electromagnetic mechanisms on the linear growth rate of the Electron Temperature Gradient (ETG) modes are shown. The presence of fast ${ }^{3} \mathrm{He}$ ions induces a strong increase $(+50 \%)$ of the ETG modes' linear growth rate, in opposition to what happens for ITG modes. The increase of the ETG modes' linear growth rate is related only to the presence of the fast ${ }^{3} \mathrm{He}$ ions and does not depend on electromagnetic effects or on the distribution function of ${ }^{3} \mathrm{He}$. The effect of fast ions can be quantified, at least its linear effect, considering the change in $\tau=Z_{e f f} T_{e} / T_{i}$ they induce. This parameter strongly affects the linear growth rate of ETG modes, as the ETG critical threshold in $R / L_{T e}$, when fixing the other plasma parameters, varies as $(1+\tau)$ [40]. In our case $\tau \approx 1.5$ with no fast ions and $\tau \approx 1$ with fast ions, inducing, in our linear simulations, a shift of the linear ETG critical threshold from $\approx 5.3$ to $\approx 4.6$, as can be seen in figure 4d. This change in the ETG linear threshold, comparable with the ITG one in presence of fast ions, and the very high ETG stiffness, lead to values of the ratio between the ETG growth rate and the ITG growth rate, at experimental conditions, higher than the electron to deuterium mass ratio, indicating favorable conditions for ETG modes.

\section{$5.2 \quad$ Nonlinear gyrokinetic simulations}

The results from the nonlinear gyrokinetic simulations and their comparison with the experiment are shown in figures $7 \mathrm{a}$ and $7 \mathrm{~b}$ for ions (using $s=0.46$ and $s=0.25$ ) and in figures $8 \mathrm{a}$ and $8 \mathrm{~b}$ for electrons (using $s=0.46$ and $s=0.25$ ). The results from the electron-scale nonlinear simulations for ETG modes are shown in figure 8c.

\subsubsection{Effects of fast ions on ion thermal transport}

When the fast ${ }^{3} \mathrm{He}$ ions are not considered, the experimental ion heat flux is strongly over predicted by the simulations, with $q_{i}^{\text {sim }} \sim 4 q_{i}^{\text {exp }}$, in both the electrostatic and in the electromagnetic simulations. In our case, electromagnetic effects without fast ions have no strong effects in the simulation. A reduction of $\sim 40 \%$ of the ion heat flux is predicted when fast ions are considered in the simulation without taking into account the electromagnetic effects. This reduction is caused by pure electrostatic effects and can be explained by mechanisms related to a wave-fast particles resonant interaction, as suggested in [9]. According to what observed in the linear simulations (figure 5), the stabilization affects mainly the ${ }^{3} \mathrm{He}$ flux. Considering also the electromagnetic effects in the simulations with fast ions, a strong stabilization of $q_{i}(-60 \%)$ is obtained. This nonlinear stabilization of the turbulent ion flux, enhanced by the fast ions pressure, strongly affects the bulk ion (D) flux. This reduction of $q_{i}$ permits to reach the experimental level of the ion heat flux. In total, a $q_{i}$ reduction of $\sim 75 \%$ is predicted when considering fast ions and electromagnetic effects and using a Maxwellian distribution function for fast ions. Using a numerical distribution function for the ${ }^{3} \mathrm{He}$ ('SELFO' in the figures), there is still a total strong reduction of the ion heat flux with respect to the case without fast ions $(-55 \%)$, but $q_{i}$ is now overestimated with respect to the experiment $(+75 \%)$. Using a numerical distribution function for the ${ }^{3} \mathrm{He}$, as observed in the previous section, avoids the presence of low- $k_{y}$ electromagnetic modes and allows to compare the results from nonlinear simulations and the experimental fluxes also for the nominal value of the magnetic shear, $s=0.25$. In this case, the experimental $q_{i}$ is well reproduced within error bars (figure $7 \mathrm{~b}$ ). 
Nonlinear simulations have been performed also for the low ICRH case (green stars in the figures). In this case both $q_{e}$ and $q_{i}$ are well reproduced within error bars. Comparing the results for the low ICRH case and for the high ICRH case, it appears that the nonlinear simulations predict a reduction of the ion stiffness when fast ions and electromagnetic effects are considered. This is in agreement with previous studies [4, 7, 8] and with past experimental observations [2]. These results clearly show that both fast ${ }^{3} \mathrm{He}$ ions and electromagnetic effects are necessary in order to reproduce the experimental level of the ion heat flux. Their effects lead to a strong stabilization of ITG modes, both linear and nonlinear, and is independent of the plasma rotation. The mechanisms that participate in the ITG turbulence stabilization are both electrostatic and electromagnetic, the last being a determinant factor in reproducing the experimental fluxes.

\subsubsection{Effect of fast ions on electron thermal transport}

The effect of fast ${ }^{3} \mathrm{He}$ on the electron heat flux is similar to the one observed for the ion heat flux (figure 8). When the fast ${ }^{3} \mathrm{He}$ ions are not considered, the experimental electron heat flux is strongly over predicted by the simulations, with $q_{e}^{\text {sim }} \sim 2.5 q_{e}^{e x p}$, while a strong reduction of $q_{e}$ is predicted when fast ions and electromagnetic effects are considered in the simulation. This is related to the strong suppression of ITG modes that causes also the strong reduction of $q_{e}$.

When the ion heat flux is matched in the simulations, the experimental electron heat flux is under-predicted $(-(30-50) \%)$. The lack of electron heat flux in the low-k electromagnetic simulations with fast ${ }^{3} \mathrm{He}$ could be due to the fact that high-k modes are more important in presence of fast ions, as suggested by the linear gyrokinetic simulations (figures $4 \mathrm{c}$ and $4 \mathrm{~d}$ ). Fast ions strongly affects our electron-scale nonlinear simulations (figure 8c). In these simulations, with $s=0.25$, the amount of $q_{e}$ due to ETG modes is $\sim 5 \%$ of the experimental value when not considering fast ions and $\sim 10 \%$ of the experimental value when the fast ions are considered $(+100 \%)$. If $s=0.46$ is used, the electron heat flux carried by ETG modes in the simulation increase to the $\sim 30 \%$ of the experimental value. These indications suggest that fast ions must be considered, at least in certain experimental conditions, for a proper study of both low-k and high-k instabilities. The role of ETG modes in the studied discharges is just hypothetical. For a proper study of these modes, multi-scale gyrokinetic simulations, requiring too much computational resources, would be needed. This study is beyond the scope of this work.

\section{Conclusions}

Experimental evidence of a strong stabilization of thermal transport when using high ICRH power in $\left({ }^{3} H e\right)-D$ minority scheme in JET ILW L-mode plasmas with low plasma rotation and low fast $D$ ion pressure has been obtained. The only substantial changes in the plasmas are related to the enhancement of the fast ${ }^{3} \mathrm{He}$ ions density energy, inside $\rho_{\text {tor }} \approx 0.4$, when high ICRH power $(\sim 7 M W)$ is applied. Linear and nonlinear gyrokinetic simulations predict that the key mechanisms able to explain the experimental observations are related to this enhancement of fast ${ }^{3} \mathrm{He}$ ion pressure. Both electrostatic and electromagnetic effects are acting to stabilize the ITG modes, leading to a reduction of the ion stiffness. The main electrostatic mechanism can be related to a resonant wave-fast particles interaction [10, 11], as suggested in reference [9], and contribute significantly in the reduction of the linear growth rate of ITG modes. The electromagnetic stabilization acts on the linear growth rate of ITG modes, but is strongly enhanced through nonlinear effects and is fundamental to reproduce the experimental results. These results demonstrate that such stabilization mechanisms are at work irrespective of the plasma rotation, which implies that they can contribute to thermal transport reduction also in low rotating ITER plasmas. Furthermore, a strong dependence of these effects on the distribution function of the fast particles has been observed, underlying the importance of considering a realistic distribution function for fast particles when modelling them. These results strongly support the role of fast particles in the stabilization 
of the thermal transport in the plasma core in some experimental conditions. Regarding the effect of fast ions on high-k instabilities, a strong destabilization of ETG modes by fast ions, that leads to a reduction of $\tau=Z_{e f f} T_{e} / T_{i}$, is predicted. Considering just single ion and single electron scale simulations, up to $\sim 30 \%$ of the electron heat flux is predicted to be caused by ETG modes. As suggested in ref. [53,54,55], when ITG modes are stabilized or close to marginal stability, ETG modes can become very important for the heat fluxes of both ions and electrons. The presence of fast ions induces a strong stabilization of ITG modes while at the same time it strongly destabilizes ETG modes. This double effect can lead to a situation where ETG modes play a major role for the heat fluxes. For this reason, considering the effects of fast ions can be essential for the study of both ion and electron-scale instabilities.

\section{Acknowledgement}

The authors are grateful to D. R. Mikkelsen for assistance and to Jonathan Citrin and Tobias Görler for precious suggestions. This research used resources of the National Energy Research Scientific Computing Center, a DOE Office of Science User Facility supported by the Office of Science of the U.S. Department of Energy under Contract No.DE--AC02--05CH11231. Part of the simulations presented in this work were performed at the HYDRA HPC system at the Max Planck Computing and Data Facility (MPCDF), Germany. We acknowledge the CINECA award under the ISCRA initiative, for the availability of high performance computing resources and support. This work has been carried out within the framework of the EUROfusion Consortium and has received funding from the Euratom research and training programme 2014-2018 under grant agreement No 633053. The views and opinions expressed herein do not necessarily reflect those of the European Commission.

\section{References}

[1] Mantica P. et al., Phys. Rev. Lett. 102, 175002 (2009).

[2] Mantica P. et al., Phys. Rev. Lett. 107, 135004 (2011).

[3] Mantica P. et al., Plasma Phys. Control. Fusion 53124033 (2011).

[4] Citrin J. et al., Phys. Rev. Lett. 111, 155001 (2013).

[5] Citrin J. et al., Nucl. Fusion 54, 023008 (2014).

[6] Maggi C. F. et al., Nucl. Fusion 50, 025023 (2010).

[7] Citrin J. et al., Plasma Phys. Control. Fusion 57, 014032 (2015).

[8] Doerk H. et al., Phys. Plasmas 22, 042503 (2015).

[9] A. Di Siena et al., accepted for publication in Nucl. Fusion, 2018. https://doi.org/10.1088/17414326/aaaf26.

[10] C. Angioni and A. G. Peeters, Phys. Plasmas 15, 052307 (2008).

[11] T. Dannert, S. Gunter, T. Hau,, F. Jenko, X. Lapillonne, and P. Lauber, Phys. Plasmas 15, 062508 (2008).

[12] Tardini G. et al., Nucl. Fusion 47, 280 (2007).

[13] G. J. Wilkie et al., "First principles of modelling the stabilization of microturbulence by fast ions", 2018. (https://arxiv.org/abs/1801.00664). 
[14] Waltz R.E., Kerbel G.D. and Milovich J., Phys. Plasmas 1, 2229 (1994).

[15] Waltz R.E., Kerbel G.D., Milovich J. and Hammett G.W., Phys. Plasmas 2, 2408 (1995).

[16] De Oliveira F. N. et al., EPS 2017, (http://ocs.ciemat.es/EPS2017PAP/pdf/P2.176.pdf).

[17] Luce T. et al., submitted for publication to Nucl. Fusion.

[18] Guo S. C. and Romanelli F., Phys. Fluids B 5 (2), 520 (1993).

[19] Migliano P., et al., Plasma Phys. Control. Fusion 55, 055003 (2013).

[20] Van Eester D., et al., Plasma Phys. Control. Fusion 51, 044007 (2009).

[21] Hedin J. et al., Nuclear Fusion 42, 527 (2002).

[22] Hellsten T. et al., Nuclear Fusion 44, 892 (2004).

[23] Challis C., et al., Nucl. Fusion 29, 563 (1989).

[24] Brix M. et al., Review of Scientific Instruments 79, 10F325 (2008).

[25] Wesson J. and Campbell D. J., 'Tokamaks', Oxford University Press, $4^{\text {th }}$ edition (2011).

[26] Grierson B. A. et al., Rev. Sci. Instr. 83, 10D529 (2012).

[27] Delabie E. et al., Plasma Phys. Control. Fusion 52, 125008 (2010).

[28] Bonanomi N. et al., Nucl. Fusion 58, 036009 (2018).

[29] Jenko F., et al., Phys. Plasmas 7, 1904 (2000).

[30] Görler T., et al., J. Comput. Phys. 230, 7053 (2011).

[31] E. A. Frieman and Liu Chen, Physics of Fluids 25, 502 (1982).

[32] Brizard A. J., Hahm T. S., Rev. Mod. Phys. 79, 421 (2007).

[33] Miller R. L., et al., Phys. Plasmas 5, 973 (1998).

[34] G. J. Wilkie et al., J. Plasma Physics 81, 905810306 (2015).

[35] A. Di Siena et al., J. Phys.: Conf. Ser. 775, 012003 (2016).

[36] Di Siena A. et al., EPS 2017, Belfast, UK. (http://ocs.ciemat.es/EPS2017PAP/pdf/P2.159.pdf).

[37] Di Siena A. et al., "Non-Maxwellian fast particle effects in gyrokinetic GENE simulations", 2017. (https://arxiv.org/abs/1802.04561).

[38] Peeters A. G. et al., Phys. Plasmas 12, 022505 (2005)

[39] Bonanomi N. et al., Nucl. Fusion 55, 113016 (2015).

[40] Jenko F. et al., Phys. Plasmas 8, 4096 (2001).

[41] Dominguez R. R. and Rosenbluth M. N., Nucl. Fusion 29, 844 (1989).

[42] Bonanomi N. et al., Nucl. Fusion 58, 026028 (2018).

[43] Porkolab M. et al., Plasma Phys. Control. Fusion 54, 124029 (2012). 
[44] Ennever P., et al., Phys. Plasmas 22, 072507 (2015).

[45] J. Weiland and A. Hirose, Nucl. Fusion 32, 151 (1992).

[46] A. Hirose, Physics of Plasmas 7, 433 (2000).

[47] W. M. Tang et al., Nucl. Fusion 20, 1439 (1980).

[48] J. Y. Kim et al., Phys. Fluids B 5 (11), 4030 (1993).

[49] Bourdelle C. et al., Nucl. Fusion 45, 110 (2005).

[50] R. Hatzky et al., Phys. Plasmas 9, 898 (2002).

[51] A. Banon Navarro et al., Phys. Plasmas 18, 092303 (2011).

[52] P. Manas et al., Phys. Plasmas 22, 062302 (2015).

[53] Görler T. and Jenko F., Phys. Rev. Lett. 100, (2008)

[54] Maeyama S. et al. PRL 114, 255002 (2015).

[55] Howard N. T. et al., Phys. Plasmas 21, (2014). 


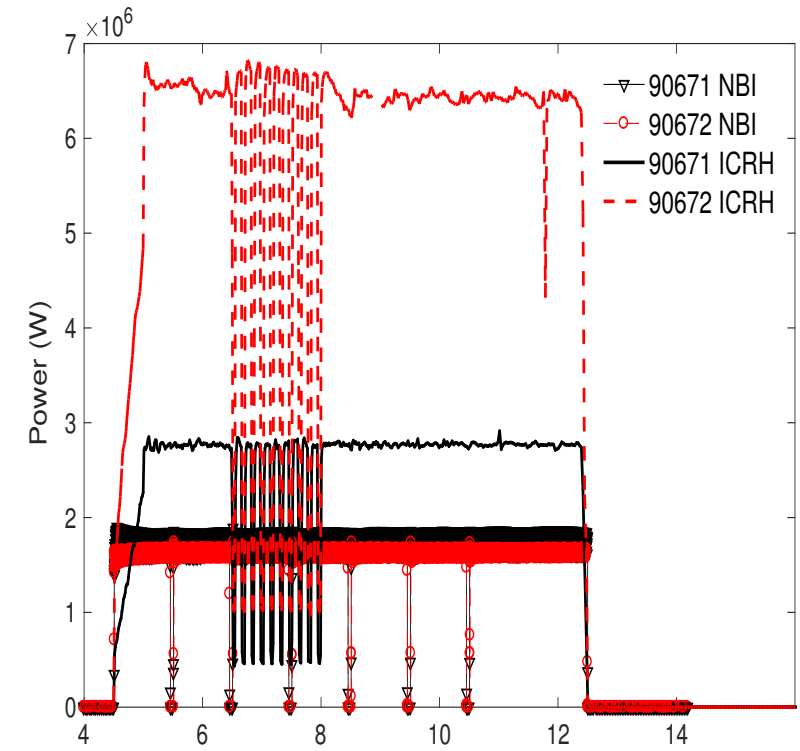

a)

$\mathrm{t}(\mathrm{s})$

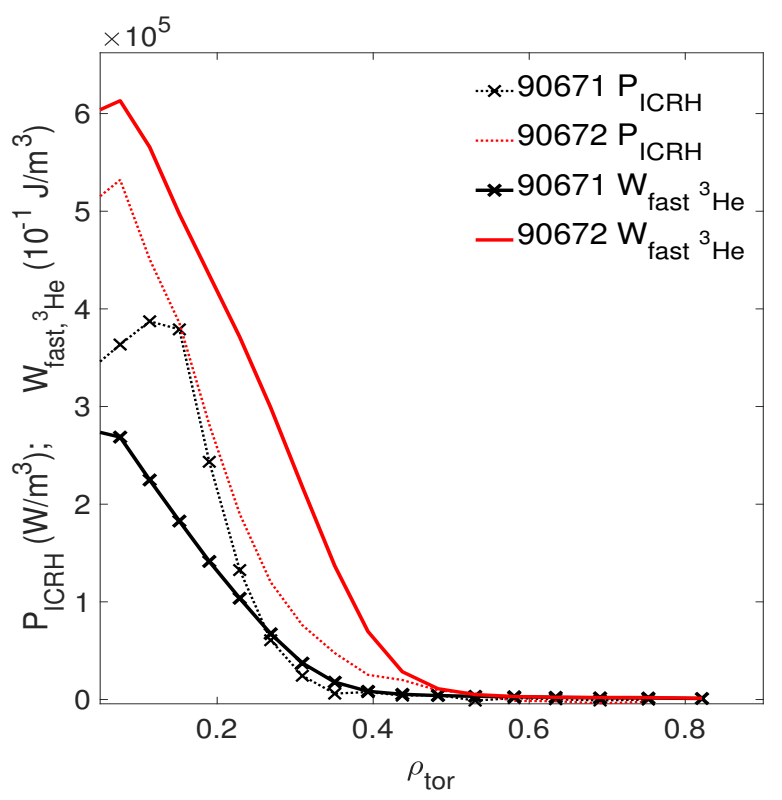

b)

Figure 1: Comparison between discharges n. 90671 and n. 90672: a) NBI and ICRH heating vs. time; b)ICRH power density to ions (dotted lines) and fast ${ }^{3} \mathrm{He}$ energy density calculated using the SELFO code.

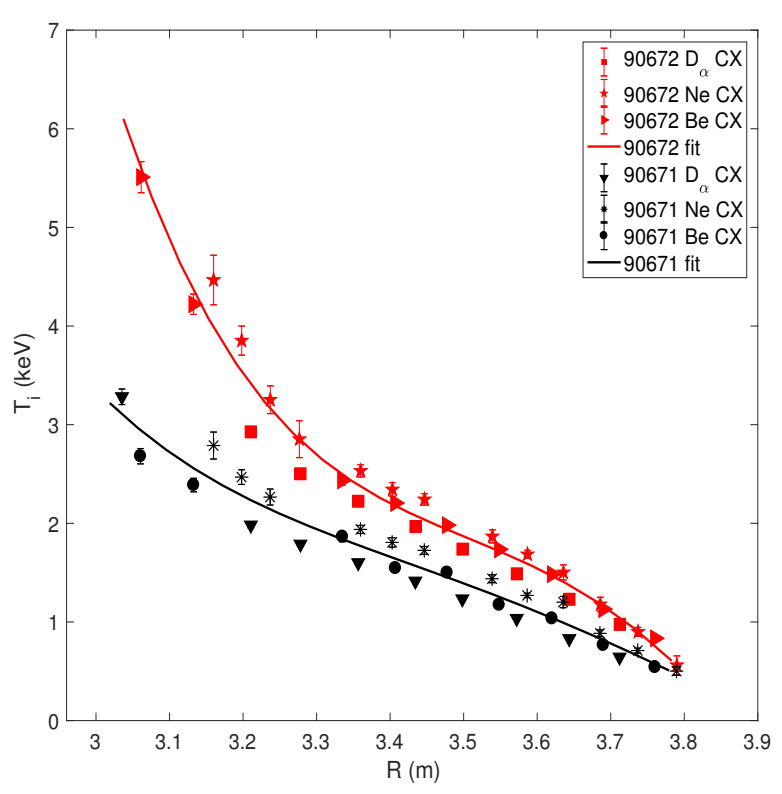

a)

Figure 2: a) Comparison between the $T_{i}$ profile of discharges n. 90671 (black squares) and n. 90672 (red circles). b) $q_{i, g B}\left(R / L_{T i}\right)$ at $\rho_{\text {tor }}=0.25$ of discharges with low ICRH power $(\sim 2.6 \mathrm{MW}$, discharges $n .90668$ and $n .90671)$ and of discharges with high ICRH power ( 6-7 MW, discharges n. 90670 and n. 90672). 


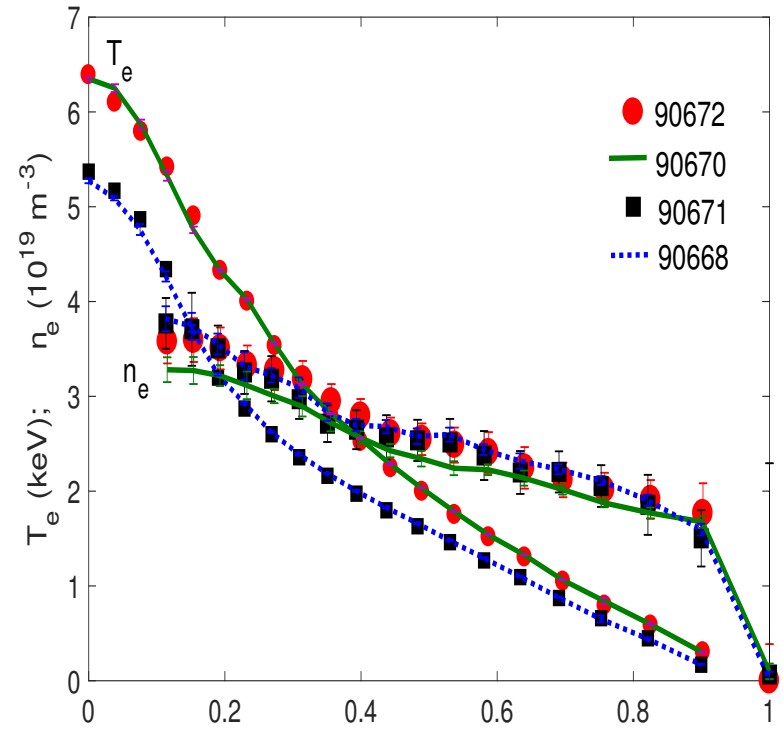

a)

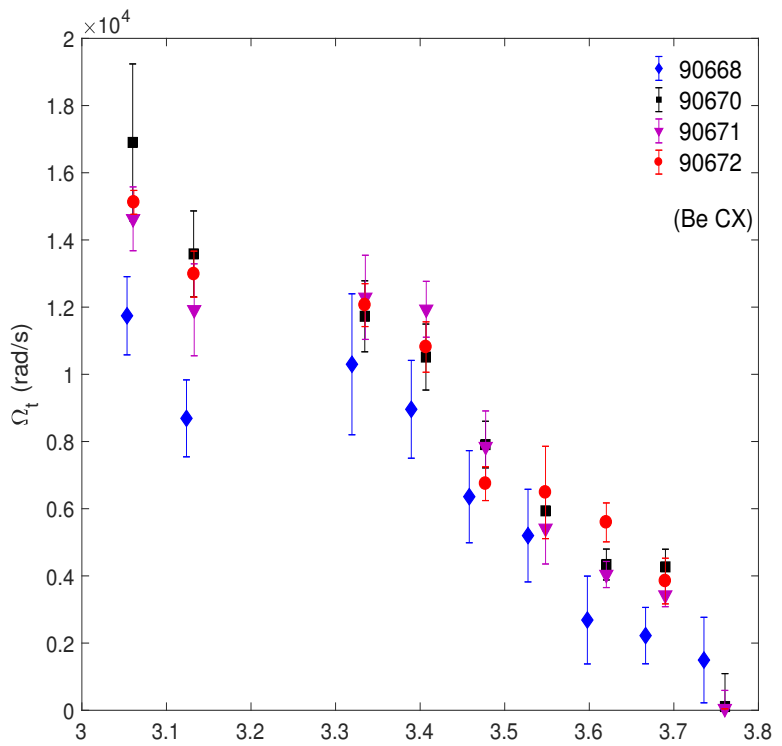

c)

$\rho_{\text {tor }}$

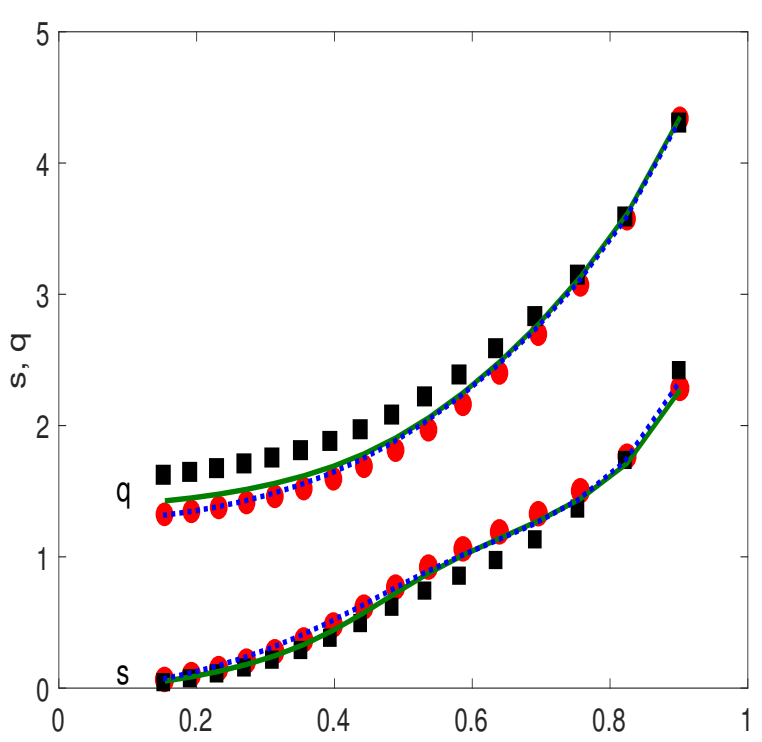

b)

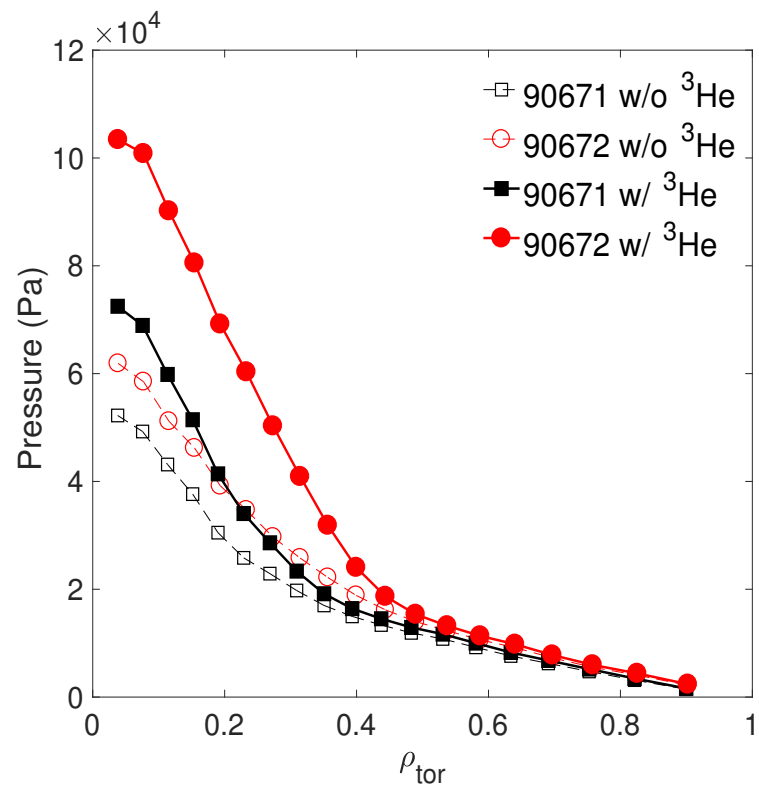

Figure 3: Comparison of radial profiles of $T_{e}, n_{e}$ (a), safety factor qand magnetic shear $s$ (b) and plasma rotation $\Omega_{t}$ (c) of discharges n. 90668 (dotted blue lines), n. 90670 (continuos green lines), n. 90671 (black squares) and n. 90672 (red circles). d) Plasma pressure without (open symbols) and with (full symbols) ${ }^{3}$ He pressure for discharges $n$. 90671 (black squares) and n. 90672 (red circles). 

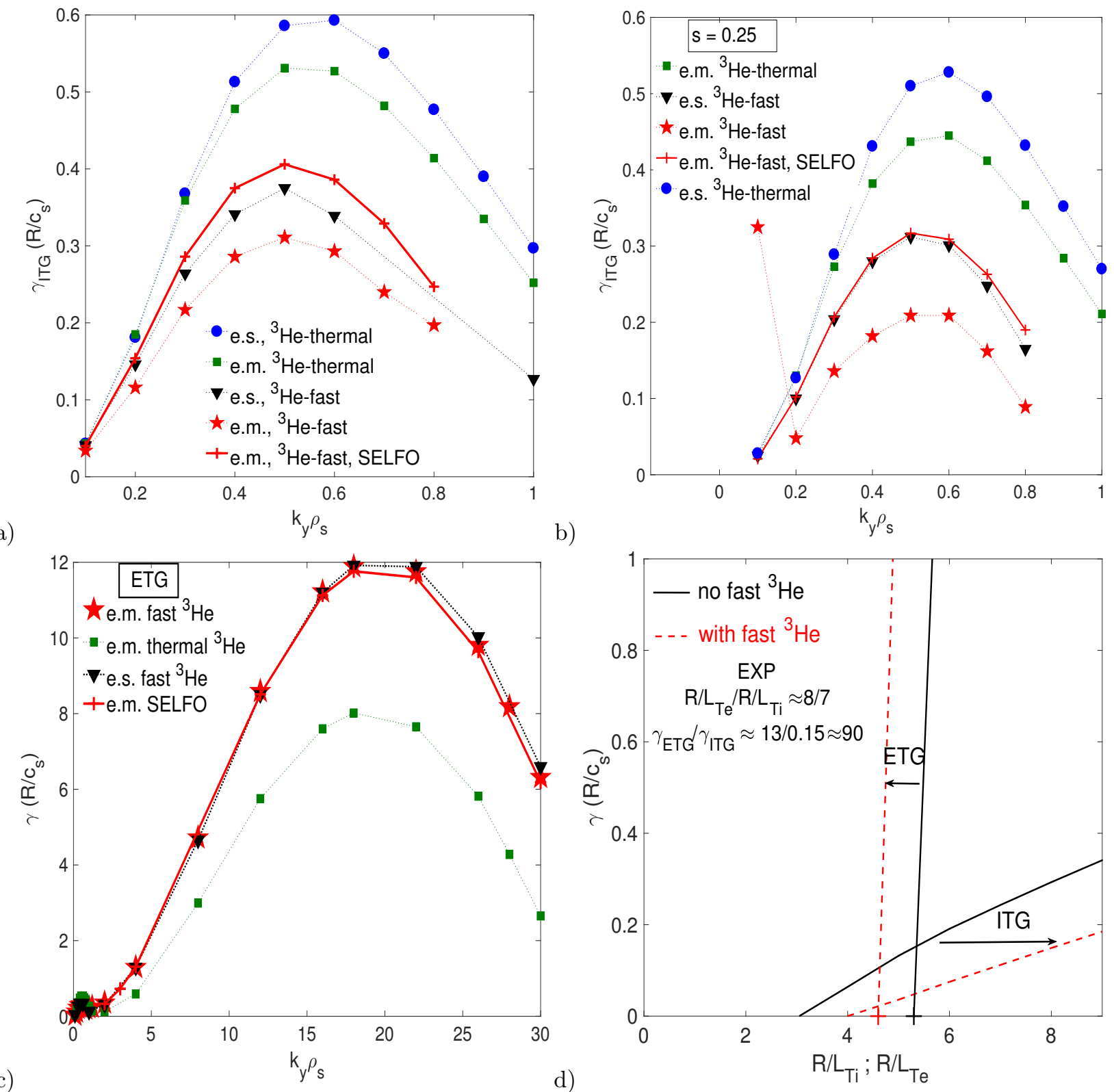

Figure 4: Linear growth rate of the most unstable mode, $\gamma\left(R / c_{s}\right)$, as a function of $k_{y} \rho_{s}$. ITG modes linear growth rate using $s=0.46$ (a) and $s=0.25$ (b), and ETG modes linear growth rate using $s=0.25$ (c). d)Comparison between the ITG (vs. $R / L_{T i}$, using $k_{y} \rho_{s}=0.3$ ) and the ETG (vs. $R / L_{T e}$, using $k_{y} \rho_{s}=20$ ) modes linear critical threshold and 'stiffness' with and without fast ${ }^{3} \mathrm{He}$ ions. 
a)
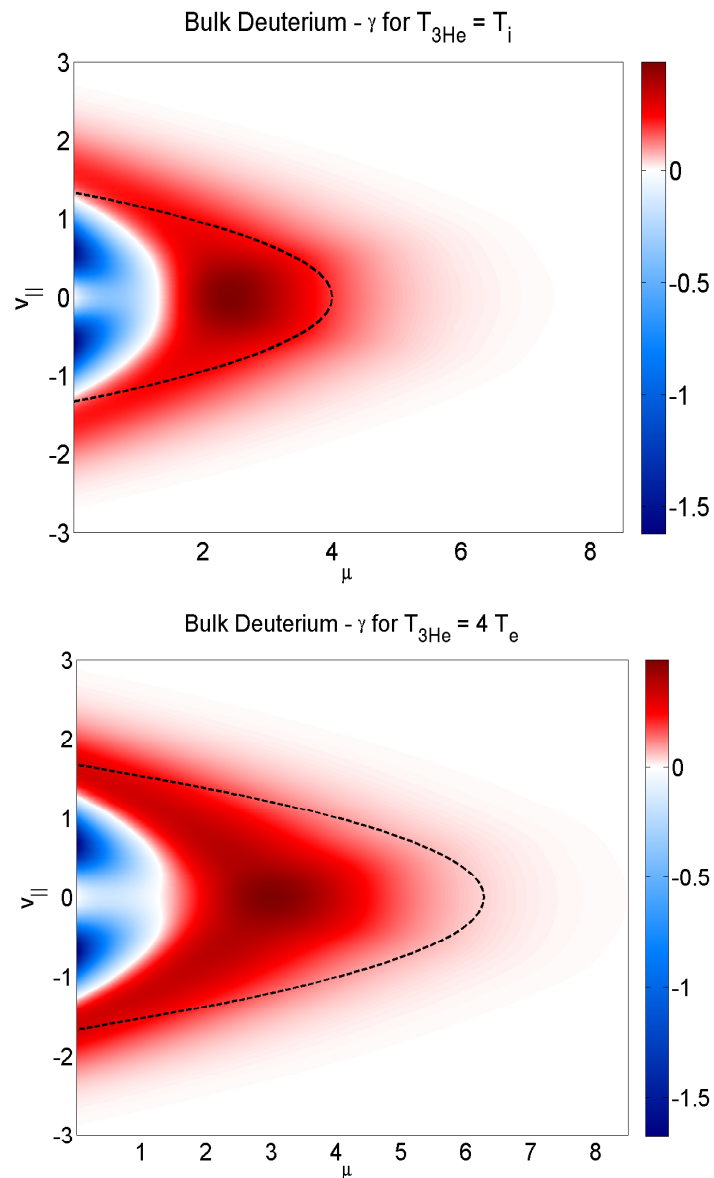

b)

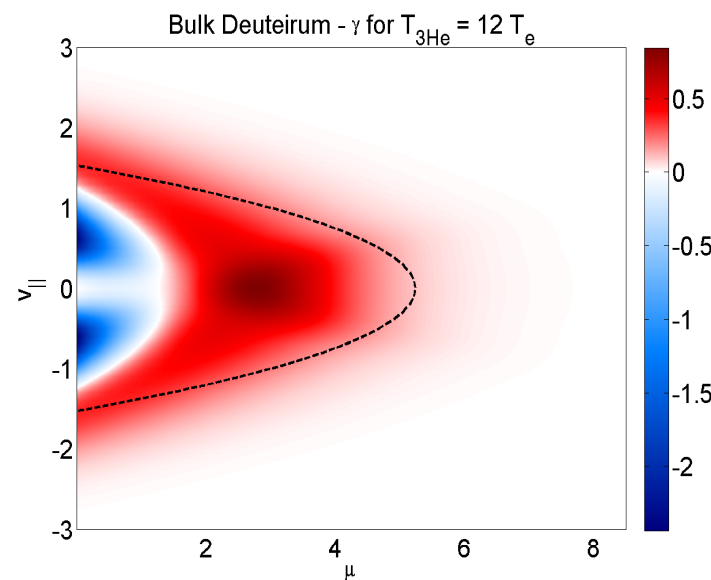

d)
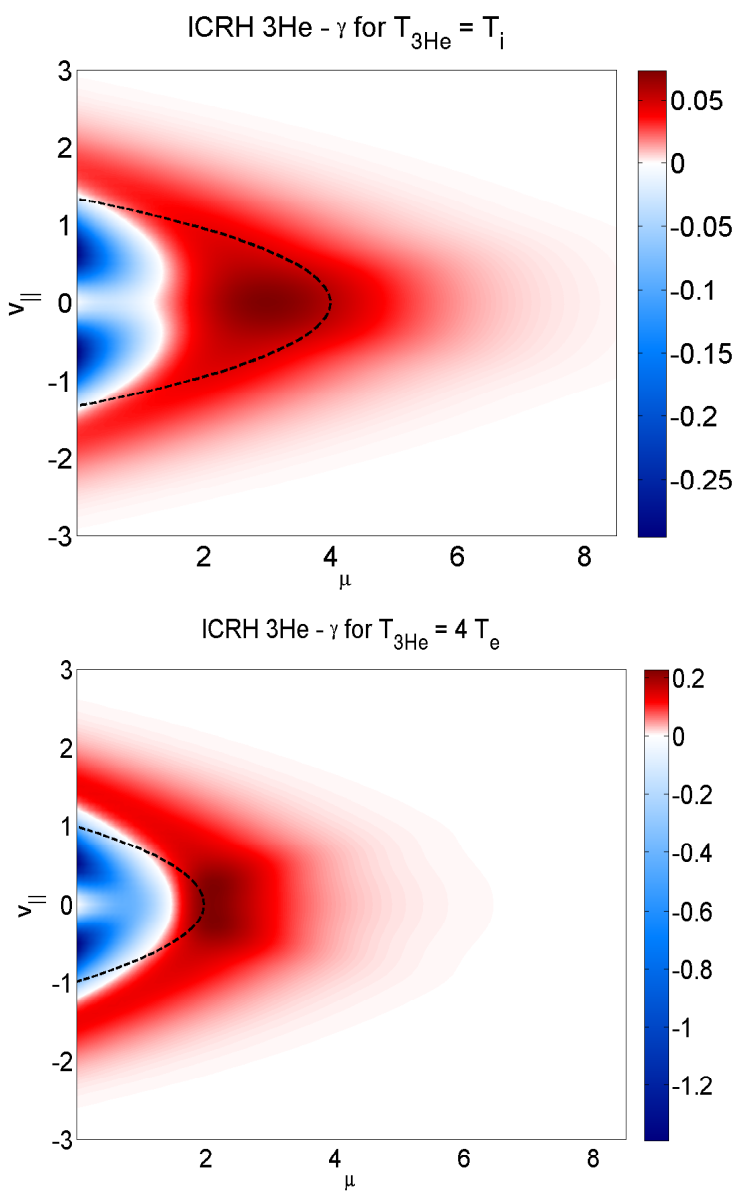

e)

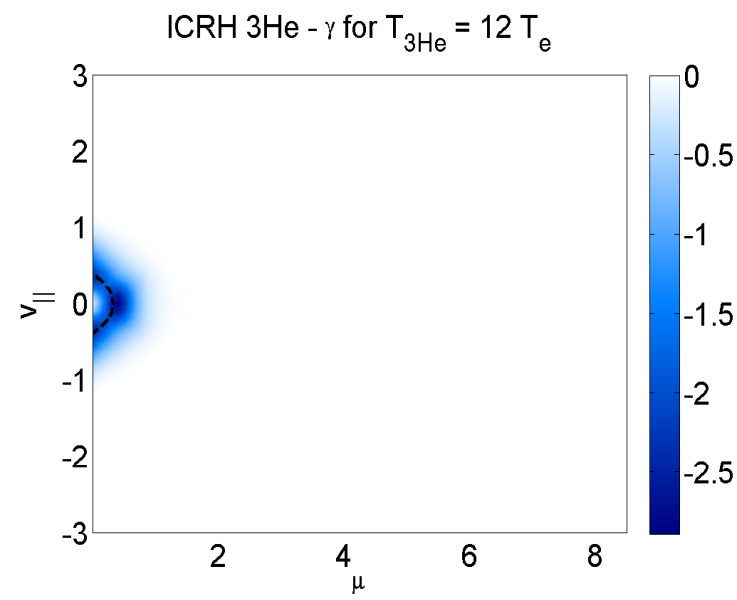

c)

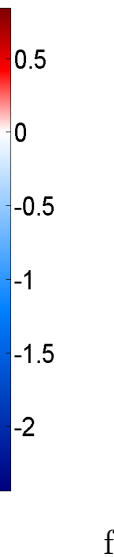

Figure 5: Converged deuterium $(a, b, c)$ and ${ }^{3} \mathrm{He}$ contribution $(d, e, f)$ to $\gamma$ velocity space structure from GENE linear simulations at low field side for the cases $T_{3_{H e}}=T_{i}$ ((a) and (d)), $T_{3_{H e}}=4 T_{e}$ (experimental low ICRH case, (b) and (e)) and $T_{3}{ }_{H e}=12 T_{e}$ (experimental high ICRh case, (c) and (f)). The black contour lines indicate the resonance positions. The value $k_{y} \rho_{i}=0.3$ has been used in the simulations. 

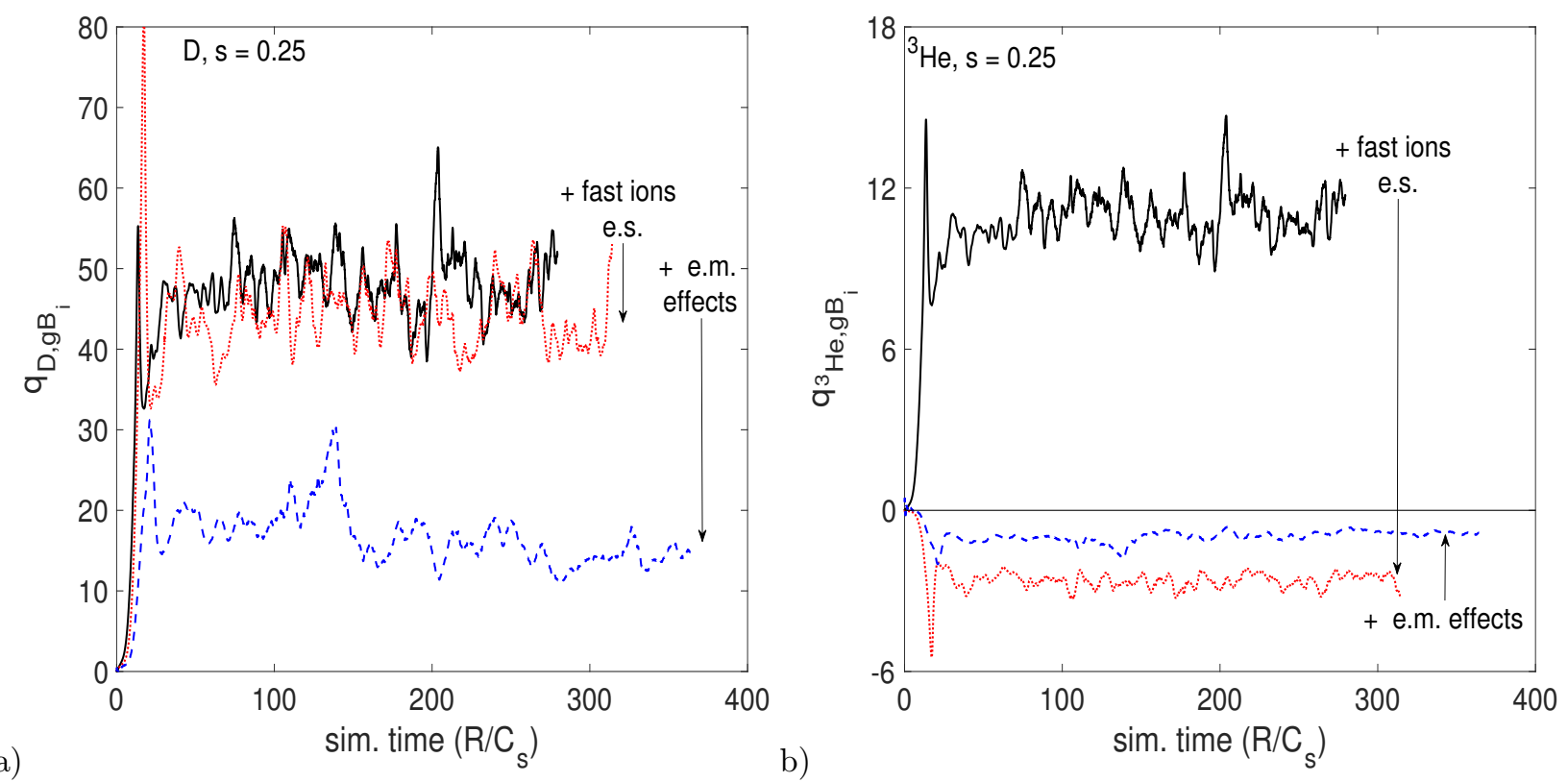

Figure 6: Time evolution of the normalized gyro-Bohm flux for $D$ (a) and ${ }^{3} \mathrm{He}$ (b) from nonlinear GENE simulations for the $s=0.25$ case.
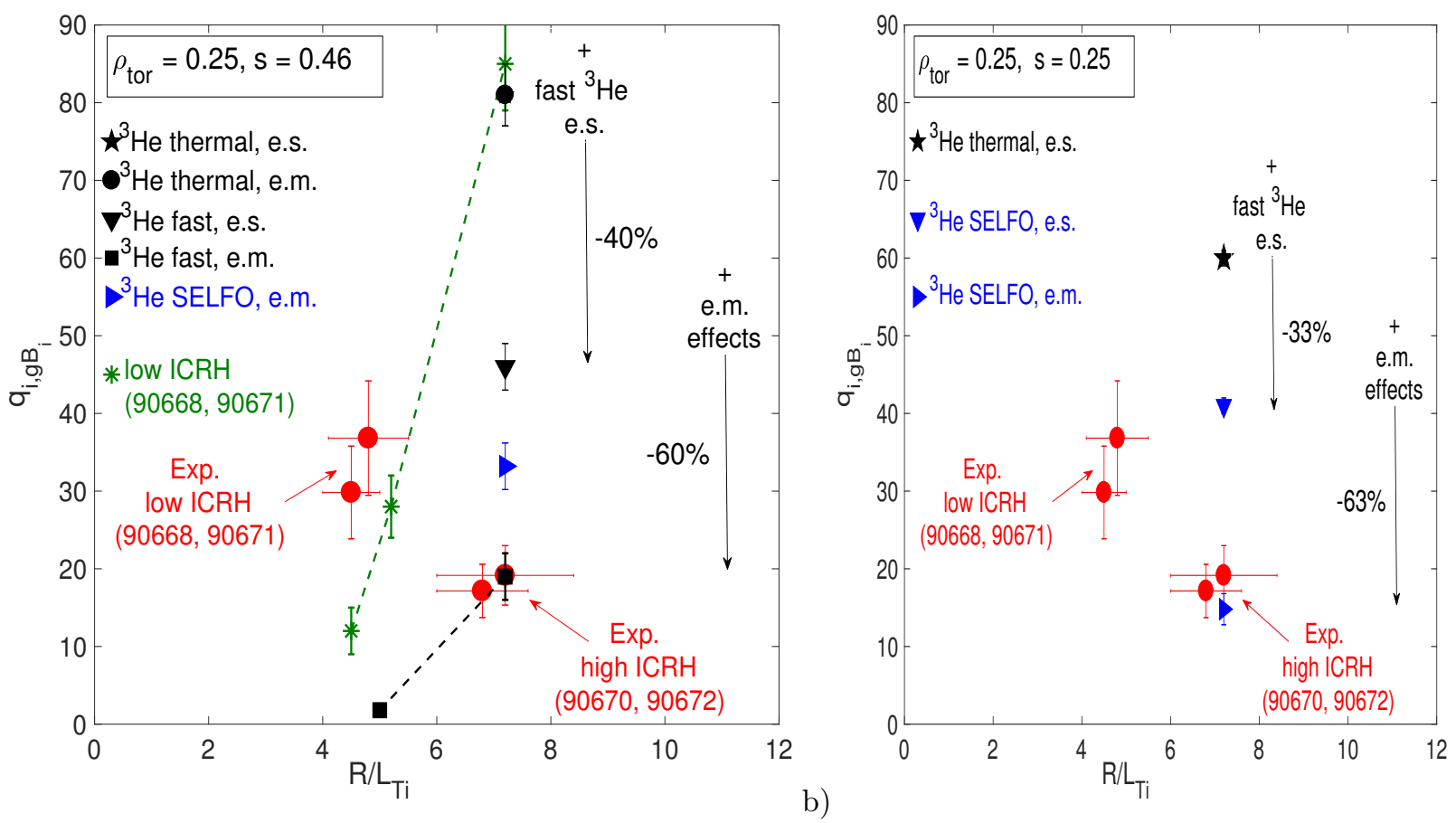

b)

Figure 7: $q_{i, g B_{i}}\left(R / L_{T i}\right)$ using $s=0.46$ (a) and $s=0.25$ (b). Comparison between nonlinear gyrokinetic simulations and experiment. 

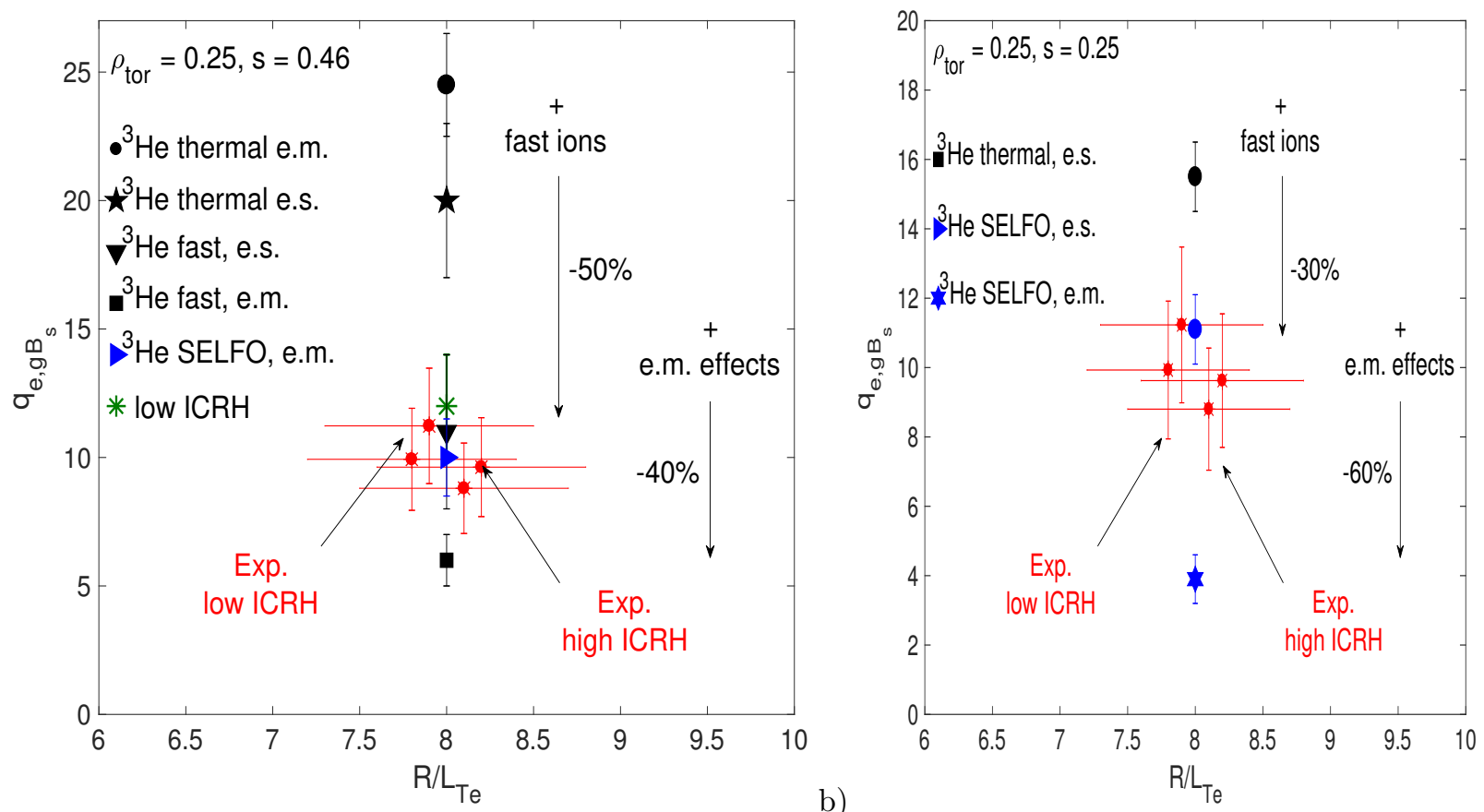

a)

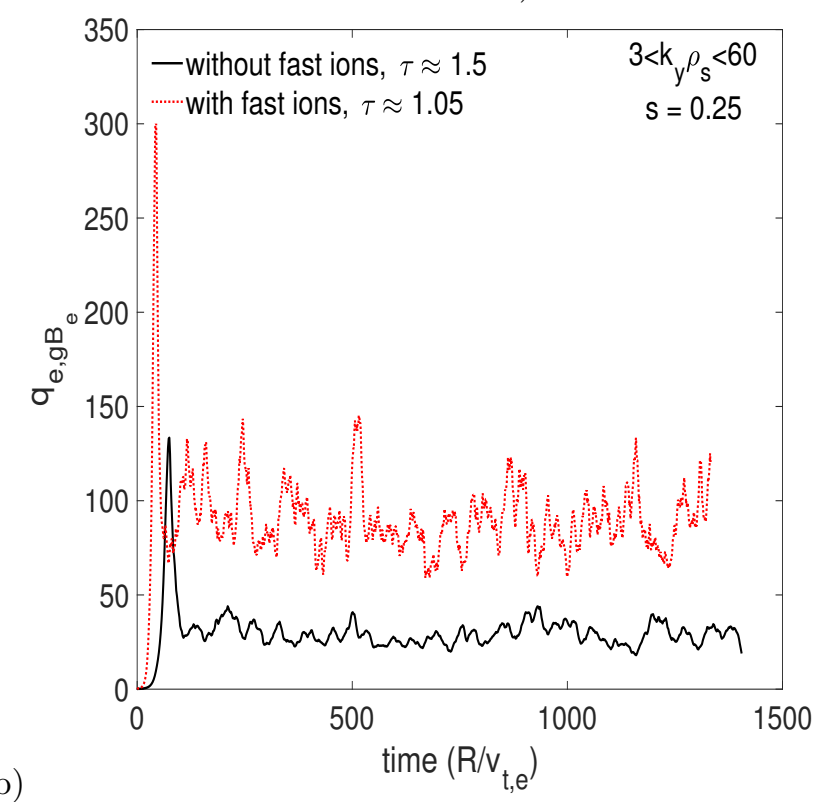

Figure 8: $q_{e, g B_{s}}\left(R / L_{T e}\right)$ using $s=0.46$ (a) and $s=0.25$ (b). Comparison between nonlinear gyrokinetic simulations and experiment. c) $q_{e, g B_{e}}$ from electron-scale simulations as a function of the simulation time (using $s=0.25$ ). 